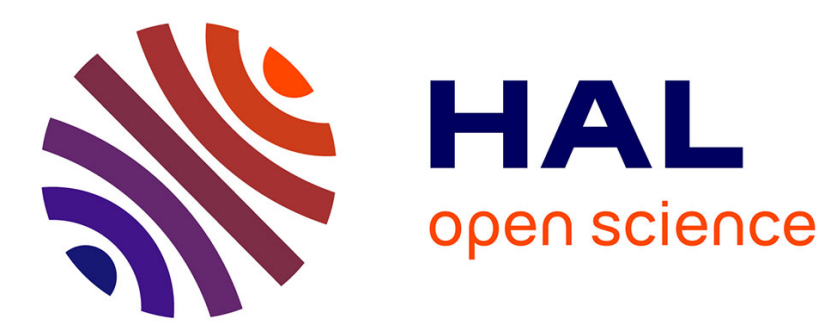

\title{
Do manufactured and natural objects evoke similar motor information? The case of action priming.
}

Marc Godard, Yannick Wamain, Solène Kalénine

\section{To cite this version:}

Marc Godard, Yannick Wamain, Solène Kalénine. Do manufactured and natural objects evoke similar motor information? The case of action priming.. Quarterly journal of experimental psychology (2006), 2019, Quarterly journal of experimental psychology (2006), 72 (12), pp.2801-2806. $10.1177 / 1747021819862210$. hal-02960030

\section{HAL Id: hal-02960030 \\ https://hal.univ-lille.fr/hal-02960030}

Submitted on 7 Oct 2020

HAL is a multi-disciplinary open access archive for the deposit and dissemination of scientific research documents, whether they are published or not. The documents may come from teaching and research institutions in France or abroad, or from public or private research centers.
L'archive ouverte pluridisciplinaire HAL, est destinée au dépôt et à la diffusion de documents scientifiques de niveau recherche, publiés ou non, émanant des établissements d'enseignement et de recherche français ou étrangers, des laboratoires publics ou privés. 


\section{Do manufactured and natural objects evoke similar motor information? The case of action priming.}

\begin{tabular}{|c|c|}
\hline Journal: & Quarterly Journal of Experimental Psychology \\
\hline Manuscript ID & QJE-STD-18-410.R2 \\
\hline Manuscript Type: & Standard Article \\
\hline $\begin{array}{r}\text { Date Submitted by the } \\
\text { Author: }\end{array}$ & 05-Jun-2019 \\
\hline Complete List of Authors: & $\begin{array}{l}\text { Godard, Marc; Univ. Lille, CNRS, UMR } 9193 \text { - SCALab - Sciences } \\
\text { Cognitives et Sciences Affectives, F - 59000, France, Psychology } \\
\text { Wamain, Yannick; Univ. Lille, CNRS, UMR 9193 - SCALab - Sciences } \\
\text { Cognitives et Sciences Affectives, F - 59000, France, Psychology } \\
\text { Kalénine, Solène; Univ. Lille, CNRS, UMR 9193 - SCALab - Sciences } \\
\text { Cognitives et Sciences Affectives, F - 59000, France, Psychology }\end{array}$ \\
\hline Keywords: & $\begin{array}{l}\text { embodied cognition, priming, action representations, object concepts, } \\
\text { semantic categorization }\end{array}$ \\
\hline
\end{tabular}




\title{
Do manufactured and natural objects evoke similar motor information?
}

\section{The case of action priming.}

Marc Godard, Yannick Wamain, Solène Kalénine*

Univ. Lille, CNRS, UMR 9193 - SCALab - Sciences Cognitives et Sciences Affectives, F 59000, France

\author{
*Corresponding author: Solène Kalénine (solene.kalenine@univ-lille.fr) \\ Univ. Lille, CNRS, UMR 9193 - SCALab - Sciences Cognitives et Sciences Affectives, F - \\ 59000, France
}

Word count total (including: abstract, main text, figures, tables, footnotes): 3639

Word count (abstract): 181

Word count (Title page): 34

Number of references: 26 references

Number of pages (without figures and tables): 17 pages 


\begin{abstract}
There is considerable evidence that visually presented manipulable objects evoke motor information, supporting the existence of affordance effects during object perception. However, most arguments come from stimulus-response compatibility paradigms, raising the issue of the automaticity of affordance effects. Action priming paradigms overcome this issue but show less reliable results, possibly because affordance effects are moderated by additional factors. The present study aimed to assess whether affordance effects highlighted in action priming paradigms could be affected by object category (manufactured or natural). Twentyfour young adults performed a semantic categorization task on natural and manufactured target objects presented after neutral (non-grasping hand postures) or action (congruent power or precision grips) primes. Results revealed a modulation of action priming effects as a function of object category. Object semantic categorization was faster after action than neutral primes, but only for manufactured objects. Results suggest that natural and manufactured objects evoke distinct types of affordances and that action priming paradigms favour the evocation of functional affordances during object semantic categorization. This finding fuels the debate on the nature of the motor information evoked by visual objects.
\end{abstract}

Keywords: embodied cognition, priming, action representations, object concepts, semantic categorization 


\section{Introduction}

The literature on visual perception highlights close connections between action and the perception and recognition of objects, in accordance with embodied and grounded views of cognition (e.g., Barsalou, 2008; Gallese \& Lakoff, 2013). In particular, motor affordance effects show that object perceptual and conceptual processing is affected by the evocation of their typical gestures (Thill, Caligiore, Borghi, Ziemke, \& Baldassarre, 2013; van Elk, van Schie, \& Bekkering, 2014, for review). At the neural level, affordances effects are supported by the activation of motor brain areas during perception and identification of manipulable objects (Gerlach, Law, \& Paulson, 2002).

Classically, the effects of object affordances on perceptual and semantic judgements are demonstrated in visuo-motor tasks (e.g., Bub, Masson, \& van Mook, 2018; Lindemann, Abolafia, Girardi, \& Bekkering, 2007; Tucker \& Ellis, 2001). In one first study, Tucker and Ellis (2001) showed activation of object motor properties during object semantic categorization using a stimulus-response compatibility paradigm. Real objects that could be small (i.e. grasped with a precision grip) or large (i.e. grasped with a power grip) were presented. Participants had to determine whether objects were natural or manufactured by grasping a device with either a precision or a power grip. Response times were faster when the response grip was compatible with the grip evoked by object real size. Results were interpreted as automatic activation of the specific grasp gestures associated with the object when accessing the object concept. Yet this interpretation faces two challenges. First, real object perception provides direct visual information about object size. Thus, affordance effects could occur without motor information being a part of the object conceptual representation. The issue has been overcome by displaying objects in a standardized size (Borghi et al., 2006; Ni, Liu, \& Yu, 2018; Yu, Abrams, \& Zacks, 2014). Second, the presence of a specific motor response questions the automaticity of affordance effects. Some authors 
have thus proposed alternative paradigms that do not involve specific motor responses to investigate affordance effects during object visual processing.

Action priming paradigms may be particularly relevant in this regard (Borghi et al., 2006; Helbig, Steinwender, Graf, \& Kiefer, 2010; Kalénine, Bonthoux, \& Borghi, 2009; Ni et al., 2018; Perraudin \& Mounoud, 2009). In one first priming study, Borghi et al. (2006) presented object pictures in their standardized size that were primed by pictures of hand postures. Each object was presented three times, once with a compatible hand posture (e.g., orange-clench), once with an incompatible hand posture (e.g., orange-pinch) and once with a non-grasping hand posture (e.g., orange-palm). Participants had to categorize target objects as manufactured or natural by pressing two different keys. When the prime displayed the palm posture, they had to refrain from responding (catch-trials). The authors reported a priming effect with shorter response times after compatible than incompatible action primes, but only when the grasp presented in the prime had been previously practiced by the participants. Although the demonstration of priming effects is promising, the presence of prior action practice weakens the impact of priming results regarding the automaticity of affordance evocation.

However, many additional factors may explain the low reliability of action priming effects. For instance, affordance effects have been shown to be modulated by the visual scene (e.g., Kalénine, Shapiro, \& Buxbaum, 2013), action intentions (e.g., Lee, Middleton, Mirma, Kalénine, \& Buxbaum, 2013), task demands (e.g., Tipper, Paul, \& Hayes, 2006), and object types (e.g., Ferri, Riggio, Gallese, \& Costantini, 2011). Interestingly, when Ferri et al. (2011) asked participants to categorize objects as natural or manufactured using compatible or incompatible reach-to-grasp movements, they found affordance effects for manufactured objects but not for natural objects. Thus, the difficulty to highlight priming effects in studies such as Borghi et al. (2006) may also be due to the mix of different object categories, some of them showing the effect and some others not. The present study aims to assess whether 
manufactured and natural objects show similar affordance effects in action priming. An adaptation of Borghi et al. (2006)'s paradigm was used. If object category accounts, at least to some extent, for the vulnerability of action priming effects, then we should observe a modulation of action priming according to object category with greater (or even exclusive) priming for manufactured objects.

\section{Method}

\section{Participants}

Twenty-four adults (mean age $=25$; age range $18-53,14$ women) took part in the experiment ${ }^{1}$. All participants were right-handed and had normal or corrected-to-normal visual acuity. The entire protocol was approved by the Ethical Committee of the University and was in accordance with the declaration of Helsinki (1964, revised in 2013). All participants gave written informed consent and were not paid for their participation.

\section{Stimuli}

Photographs of manipulable objects were selected from open source database (Pixabay). Object size was standardized. Objects were displayed in a fictive square of 500 x 500 pixels on a black background centered on their horizontal axis. Among the 50 objects selected (40 for test-trials +4 for catch-trials +6 for practice-trials), 25 were manufactured (e.g., bowl) and 25 were natural (e.g., apple). In each category, half of the objects were usually grasped with a precision grip (e.g., cherry, marble) and half were usually grasped with a power grip (e.g., apple, bowl) according to their real size. The 40 test object pictures are presented in Appendix 1.

1 "Sample size was determined using the power.t.test function of the pwr R package considering a medium effect size and a statistical power of 0.80 for the expected priming advantage for artifact vs. natural objects". 
Photographs of a hand in five different postures were designed and displayed on a black background. The center of the hand was placed in the middle of the screen. Among the five hand pictures, twice displayed a grasping hand posture (power or precision grip) and three displayed a non-grasping hand posture (palm-up, palm-down and fist). All pictures were displayed on a 27" screen $(1920$ x 1080 pixels, $120 \mathrm{~Hz})$ with MatLab 9.2 (MathWorks, Natiek, USA) and Psychophysics Toolbox extensions.

\section{Controls of the stimuli}

Thirteen additional participants judged the overall manipulability and the variability of manipulation of the 40 objects used the experiments presented among 40 fillers selected from Salmon, McMullen and Filliter (2010). They rated on a 5-point Likert scale a) "the manipulability of the object according to how easy it is to grasp and use the object with one hand" and b)" the extent to which the way you manipulate the object can vary for each manipulation". Natural and artifact objects were judged highly and equally manipulable (median $=5$ [range of value: 5 to 5 ] for natural objects and median: 5 [range: 5 to 5] for manufactured objects). Moreover, natural and artefact objects showed low and equivalent variability in the way they are manipulated (median $=2$ [range of value: 1 to 2 ] for natural objects and median: 2 [range of value: 1 to 3] for manufactured objects).

\section{Procedure}

Participants were seated $60 \mathrm{~cm}$ from the screen. Stimuli were inserted in an action priming paradigm with hand pictures as primes and object pictures as targets. Each trial started with a fixation cross presented at the center of the screen for $500 \mathrm{~ms}$ followed by one of the hand primes for $500 \mathrm{~ms}$ (Figure 1). Then the object target was presented until participants' response or for a maximum of $4000 \mathrm{~ms}$. Participants were asked to categorize the object as natural or manufactured by pressing the "q" and "m" keys of an Azerty keyboard with their left and right hand. Response mapping was counterbalanced between participants. Response times and errors were recorded. 
During the test phase, each target object was presented twice, once with the appropriate grasping hand prime (power or precision grip) in the action priming condition and once with one of two non-grasping hand primes (palm-down or fist) in the neutral priming condition, leading to 80 experimental trials. Eight additional catch-trials (10\%) were designed using four additional target objects presented with the palm-up "no-go" prime. On catch-trials, participants were asked to refrain from responding in order to ensure that they paid attention to the primes. The 88 trials were randomly presented.

Participants performed 20 representative practice trials beforehand involving six additional target objects.

\section{--- Insert Figure 1 about here ---}

\section{Results}

Performance on catch-trials was verified for each participant (mean accuracy $=93 \%$, $\min =$ $63 \%$ ) but analyses were restricted to critical trials. Response times (RTs) inferior to $200 \mathrm{~ms}$ and superior to $3000 \mathrm{~ms}(1 \%)$ and RTs for incorrect responses (3\%) were excluded from the analyses. RTs were finally trimmed by removing those exceeding 3 standard deviations from the participant's mean in each condition. Overall, $3.13 \%$ of RTs were excluded $(3.96 \%$ for neutral priming x manufactured objects; $4.38 \%$ for action priming $\mathrm{x}$ manufactured objects, $2.71 \%$ for neutral priming $\mathrm{x}$ natural objects and $2.08 \%$ for action priming x manufactured objects). Mean RTs and standard deviations in the different conditions are reported in Table 1 and raw data are available here https://osf.io/8qzkc/?view only=4f0b14f72e3940f38bfff87bfa2499c0. Logarithmic transformation was applied on RTs. Visual inspection of the distribution of residuals after logtransformed did not show important deviations from normality. 
A mixed-effect model was used to analyze log transformed RTs as a function of Priming (action, neutral) and Category (natural, manufactured). Mixed-effect-linear models do not require prior averaging of the data and allow taking into account differences between individuals and variation in their sensitivity to the factors of interest (Baayen, Davidson, \& Bates, 2008; "random intercepts" and "random slopes", see below). In the present experiment, there were 24 subjects $\times 20$ items $=480$ RTs in each Priming $\mathrm{x}$ Category condition. We also added Grasp type (power and precision grip) to the model, as it has been shown to influence RTs (Borghi et al., 2006). Thus, fixed effects corresponded to the effect of Priming, Category, Grasp Type and their interactions. We predicted possible main effects of Priming, Category, Grasp Type and a Category x Grasp Type interaction but more critically, we excepted an interaction between Priming and Category. The random effect structure of the model included participants as random effect factor with random intercepts and random slopes for priming (analyses conducted with lme4 3.0-1 package of $\mathrm{R}$ version 3.4.4).

\section{--- Insert Table 1 about here ---}

LmerTest package (version 3.0-1) was used to obtain significance F-tests of fixed effects. Denominator degrees of freedom were approximated using Satterthwaite's approximations. A R-squared value for the whole model was provided using the r.squaredGLMM function of the MuMIn package (version 1.42.1). Cohen $\mathrm{d}$ effect sizes were computed for relevant t-tests as the ratio between the estimated mean difference between conditions and the square root of the sum of the residual variance and the variance of the random effects of the model, following Westfall, Kenny and Judd (2014). 
The entire linear model explained $37 \%$ of variance $\left(\mathrm{R}^{2}=0.37\right)$. There was a main effect of Category $[\mathrm{F}(1,1826.02)=13.1, \mathrm{p}=0.001]$. RTs were shorter for natural than for manufactured objects (estimate [manufactured - natural] $=+1.7 \times 10-2, \mathrm{SE}=0.498 \times 10-2, \mathrm{~d}$ $=0.13$ ). Importantly, we observe a main effect of Priming $[F(1,671.31)=9.91, p=0.002]$. RTs were $26 \mathrm{~ms}$ shorter for correct than neutral action priming (estimate [neutral - action] = $+1.63 \times 10-2, \mathrm{SE}=0.5 \times 10-2, \mathrm{~d}=0.12$ ). There was no main effect of Grasp type $[\mathrm{F}(1,1826.03)=0.68, \mathrm{p}=0.41]$ but a marginal two-way interaction between object Grasp type and Category $[\mathrm{F}(1,1826.02)=3.8, \mathrm{p}=0.053]$. The advantage of natural $(\mathrm{n})$ objects over manufactured $(\mathrm{m})$ objects tended to be greater for objects grasped with a power grip compared to precision grip (estimate $[(m-n)$ power grip $-(m-n)$ precision grip $=+1.9 \times 10-2$, $\mathrm{SE}=0.957 \times 10-2, \mathrm{~d}=0.14)$. The predicted interaction between Priming and Category was significant $[\mathrm{F}(1,1826.02)=4.2, \mathrm{p}=0.041]$. As expected, the interaction was due to the presence of a priming effect for manufactured objects [estimate $=+1.4 \times 10-2, \mathrm{t}=3.66, \mathrm{SE}=$ $0.7 \times 10-2, \mathrm{p}=0.001, \mathrm{~d}=0.20$ ] but not for natural objects [estimate $=+0.2 \times 10-2, \mathrm{t}=0.79$, $\mathrm{SE}=0.7 \times 10-2, \mathrm{p}=0.43, \mathrm{~d}=0.04]$. No other effect was significant.

\section{Discussion}

The present experiment confirms that the evocation of motor information associated with manipulable objects affects their semantic processing. Results showed that overall, object semantic categorization was faster after action primes than neutral primes. Specifically, the presentation of typical grasping postures facilitated object categorization, even when displayed in a standardized size that does not provide direct information about the appropriate grip aperture. Furthermore, the priming paradigm did not involve the execution of specific 
grasping responses, reinforcing the idea that grasping postures may be evoked in the absence of specific motor plan. The functional role of motor information in object concepts is still largely debated (Mahon \& Caramazza, 2008). In this context, the present results support the hypothesis that some motor attributes evoked by visual objects, along with other perceptual and non-perceptual features, are integrated to object conceptual representations, in line with embodied and grounded views of concepts (e.g., Barsalou, 2008). Critically, the present experiment further demonstrates that the facilitative effect of action priming on object semantic categorization is modulated by object category. Overall, semantic categorization was faster for natural than artifact objects, consistent with previous priming studies and with an interpretation of category-specific deficits related to the higher structural similarity among natural than artifact objects (Gerlach, 2017). Yet natural objects did not benefit from action priming. In contrast, results showed that the advantage of action primes over neutral primes was driven by manufactured objects, and was actually not present for natural objects. The difference of action priming effects between object categories could not be accounted for by differences in overall manipulability or variability in object manipulation between natural and artifact object categories, since natural objects were judged as highly manipulable and as steady in their manipulation as artifact objects. The methodological and theoretical consequences of this distinction are discussed below.

Although Borghi et al. (2006) used a similar priming protocol, their priming results were not as clear-cut. In particular, they did not succeed to highlight action priming effects when the participants had no motor practice with the hand primes. The need of motor practice nuances the role of affordances in object concepts and may be a drawback for strong embodied views. At least, it suggests that action priming effects are not very robust. In the light of the present results, one possibility is that action priming effects in Borghi et al. (2006) are minimized with the use of different object categories. However, the authors did not report any 
modulation of the action priming effect by object category. The discrepancy may therefore originate from methodological choices. First, Borghi et al. (2006)'s protocol involved more object repetitions, which may have reduced the impact of action primes on target processing. Second, priming effects were computed using different baselines: incongruent action primes in their study (e.g., precision grip-orange) and neutral non-action primes (e.g., fist-orange) here. A more continuous activation of action information may have limited the activation of object-specific motor information (e.g., power or precision grip) on a trial basis and diminish the priming effect amplitude. In Borghi et al. (2006)'s experiment 2, motor practice may have decreased the impact of repetition by increasing attention to the primes and trained the motor system to differentiate the two specific grasping postures, facilitating the emergence of priming effects. Consistent with this explanation, facilitative action priming effects have been observed with dynamic action primes (Vainio, Symes, Ellis, Tucker, \& Ottoboni, 2008). In this study, participants performed a similar natural/manufactured semantic categorization task on object pictures that were preceded by several frames of the grasping movement. Although it benefited to action priming, important motor simulation of the action primes was not sufficient to highlight a modulation of action priming effect by object category in none of the studies. One interpretation is that it changes the nature of the motor information evoked by hand primes. The theoretical interpretation of the category effects proposed below might be consistent with this possibility.

Theoretically, the impact of object category on action priming effects is highly relevant as it addresses the critical issue of the nature of the motor information evoked during conceptual processing of visual objects. Recent development of affordance theories have proposed a distinction between several types of affordances (Borghi \& Riggio, 2015; Buxbaum \& Kalénine, 2010). If natural and manufactured objects evoke distinct types of affordances and this type of action priming paradigm favours the perception of one type of affordances over 
the other, then action priming effects should be differentially visible depending on object category. Borghi and Riggio (2015) disentangled stable from variable affordances. Stable affordances correspond to affordances that are not context-dependent, such as those related to object usual size. Variable affordances correspond to affordances that are context-dependent, such as those related to object orientation or location in space. In a similar line, Buxbaum and Kalénine (2010) distinguished functional from structural affordances. Functional affordances correspond to the evocation of the gestures associated with the typical use of the object (such as when manipulating the object according to its typical function). In contrast, structural affordances correspond to the evocation of the gestures associated with the typical grasp of the object (such as when picking-up the object according to its structural shape). The definition of functional affordances may overlap to a certain extent with that of stable affordances above, although it may be more restrictive: all functional affordances should be stable but not all stable affordances are functional (e.g., size-related motor information).

In the light of these recent distinctions, we suggest that the type of affordances evoked in the present action priming paradigm using hand primes are stable, and more specifically functional affordances. As natural objects are usually not associated with specific use actions, functional affordances are the privilege of manufactured objects. Thus, the presence of category-selective action priming effects indicates that accessing object semantic involves the evocation of functional - but not structural - affordances. In other words, results suggest that the information related to how picking-up the object (structural affordance) does not participate in object semantic categorization while motor information related to how using the object (functional affordance) does, leading to a different action priming effects for natural and artifact object categories. This interpretation is consistent with the very recent priming results reported by $\mathrm{Ni}$ et al. (2018). Although Ni et al. (2018) did not directly compare priming effects between natural and manufactured categories, they showed that naming 
manipulable objects affording both functional and structural actions (e.g., a gun affording trigger and power grip actions) is facilitated by functional action primes but not structural action primes. The present results reflect the consequences of the functional/structural distinction between affordances on the relation between action and object semantic categories. It further suggests that functional affordances are not only critical for accessing single concepts of manufactured objects but also for accessing their superordinate category, despite previous evidence of weaker facilitative effects of action priming on superordinate compared to basic-level categorization (Kalénine et al., 2009). The superordinate categorization of manufactured objects would rely in part on the perception of their functional affordances, a phenomenon that may induce an additional cost. This cost may be reduced by the prior activation of functional gesture representations.

To conclude, we report facilitative action priming effects on object semantic categorization. Importantly, the effect was driven by manufactured objects and was not present for natural objects. The modulation of action priming by object category is consistent with recent distinctions between affordances and suggests that the paradigm assesses the evocation of functional affordances during object semantic categorization. Action priming paradigms may thus be a promising direction to refine affordance theories (e.g., Thill et al., 2013) while taking into account the different nature of the motor information evoked by natural and manufactured object categories.

\section{Acknowledgments}

This work was funded by the French National Research Agency (ANR-16-CE28-0003 and ANR-11-EQPX-0023), supported by European funds (FEDER SCV-IrDIVE) and benefited from a regional fellowship (Hauts-de-France) to M. Godard.

\section{Conflict of interest statement}


The authors declare no conflict of interest.

\section{Supplementary Material}

The Supplementary Material is available at: qjep.sagepub.com

\section{Open Practices}

The data from the present experiment are publicly available at the Open Science Framework website: https://osf.io/8qzkc/?view_only=4f0b14f72e3940f38bfff87bfa2499c0

\section{References}

Baayen, R. H., Davidson, D. J., \& Bates, D. M. (2008). Mixed-effects modeling with crossed random effects for subjects and items. Journal of Memory and Language, 59(4), 390412. https://doi.org/10.1016/j.jml.2007.12.005

Barsalou, L. W. (2008). Grounded Cognition. Annual Review of Psychology, 59(1), 617-645. https://doi.org/10.1146/annurev.psych.59.103006.093639

Borghi, A. M., Nicoletti, R., Ricciardelli, P., Bonfiglioli, C., Lugli, L., \& Rubichi, S. (2006). Are visual stimuli sufficient to evoke motor information? Neuroscience Letters, 411(1), 17-21. https://doi.org/10.1016/j.neulet.2006.10.003

Borghi, A. M., \& Riggio, L. (2015). Stable and variable affordances are both automatic and flexible. Frontiers in Human Neuroscience, 9(June), 1-16. https://doi.org/10.3389/fnhum.2015.00351

Bub, D. N., Masson, M. E. J., \& van Mook, H. (2018). Switching between lift and use grasp actions. Cognition, 174, 28-36. https://doi.org/10.1016/j.cognition.2018.01.013

Buxbaum, L. J., \& Kalénine, S. (2010). Action knowledge, visuomotor activation, and embodiment in the two action systems. Annals of the New York Academy of Sciences, 1191, 201-218. https://doi.org/10.1111/j.1749-6632.2010.05447.x 
Ferri, F., Riggio, L., Gallese, V., \& Costantini, M. (2011). Objects and their nouns in peripersonal space. Neuropsychologia, 49(13), 3519-3524. https://doi.org/10.1016/j.neuropsychologia.2011.09.001

Gallese, V., \& Lakoff, G. (2013). The brain's concepts: The role of the sensory-motor system in conceptual knowledge. Cognitive Neuropsychology, 22(3-4), 455-479. https://doi.org/http://dx.doi.org/10.1080/02643290442000310

Gerlach, C. (2017). Normal and abnormal category-effects in visual object recognition: A legacy of Glyn W. Humphreys. Visual Cognition, 25(1-3), 60-78. https://doi.org/10.1080/13506285.2016.1258022

Gerlach, C., Law, I., \& Paulson, O. B. (2002). When action turns into words. Activation of motor-based knowledge during categorization of manipulable objects. Journal of Cognitive Neuroscience, 14(8), 1230-1239. https://doi.org/10.1162/089892902760807221

Helbig, H. B., Steinwender, J., Graf, M., \& Kiefer, M. (2010). Action observation can prime visual object recognition. Experimental Brain Research, 200(3-4), 251-258. https://doi.org/10.1007/s00221-009-1953-8

Kalénine, S., Bonthoux, F., \& Borghi, A. M. (2009). How action and context priming influence categorization: A developmental study. British Journal of Developmental Psychology, 27(3), 717-730. https://doi.org/10.1348/026151008X369928

Kalénine, S., Shapiro, A. D., \& Buxbaum, L. J. (2013). Dissociations of action means and outcome processing in left-hemisphere stroke. Neuropsychologia, 51(7), 1224-1233. https://doi.org/10.1016/j.neuropsychologia.2013.03.017

Lee, C.-L., Middleton, E., Mirma, D., Kalénine, S., \& Buxbaum, L. J. (2013). Incidental and contrext-responsive activation of structure- and function-based action features during 
object identification, 39(9), 257-270. https://doi.org/doi:10.1037/a0027533

Lindemann, O., Abolafia, J. M., Girardi, G., \& Bekkering, H. (2007). Getting a Grip on Numbers: Numerical Magnitude Priming in Object Grasping. Journal of Experimental Psychology: Human Perception and Performance, 33(6), 1400-1409. https://doi.org/10.1037/0096-1523.33.6.1400

Mahon, B. Z., \& Caramazza, A. (2008). A critical look at the embodied cognition hypothesis and a new proposal for grounding conceptual content. Journal of Physiology Paris, 102(1-3), 59-70. https://doi.org/10.1016/j.jphysparis.2008.03.004

Ni, L., Liu, Y., \& Yu, W. (2018). The dominant role of functional action representation in object recognition. Experimental Brain Research, 1-13. https://doi.org/10.1007/s00221018-5426-9

Perraudin, S., \& Mounoud, P. (2009). Contribution of the priming paradigm to the understanding of the conceptual developmental shift from 5 to 9 years of age. Developmental Science, 12(6), 956-977. https://doi.org/10.1111/j.14677687.2009.00847.x

Salmon, J. P., McMullen, P. A., \& Filliter, J. H. (2010). Norms for two types of manipulability (graspability and functional usage), familiarity, and age of acquisition for 320 photographs of objects. Behavior Research Methods, 42(1), 82-95. https://doi.org/10.3758/BRM.42.1.82

Thill, S., Caligiore, D., Borghi, A. M., Ziemke, T., \& Baldassarre, G. (2013). Theories and computational models of affordance and mirror systems: An integrative review. Neuroscience and Biobehavioral Reviews, 37(3), 491-521. https://doi.org/10.1016/j.neubiorev.2013.01.012

Tipper, S. P., Paul, M. A., \& Hayes, A. E. (2006). Vision-for-action: The effects of object 
property discrimination and action state on affordance compatibility effects.

Psychonomic Bulletin and Review, 13(3), 493-498. https://doi.org/10.3758/BF03193875

Tucker, M., \& Ellis, R. (2001). The potentiation of grasp types during visual object categorization. Visual Cognition, 8(6), 769-800.

https://doi.org/10.1080/13506280042000144

Vainio, L., Symes, E., Ellis, R., Tucker, M., \& Ottoboni, G. (2008). On the relations between action planning, object identification, and motor representations of observed actions and objects. Cognition, 108(2), 444-465. https://doi.org/10.1016/j.cognition.2008.03.007

van Elk, M., van Schie, H., \& Bekkering, H. (2014). Action semantics: A unifying conceptual framework for the selective use of multimodal and modality-specific object knowledge. Physics of Life Reviews, 11(2), 220-250. https://doi.org/10.1016/j.plrev.2013.11.005

Westfall, J., Kenny, D. A., \& Judd, C. M. (2014). Statistical Power and Optimal Design in Experiments in Which Samples of Participants Respond to Samples of Stimuli. Journal of Experimental Psychology, 143(5), 2020-2045. https://doi.org/10.1037/xge0000014

Yu, A. B., Abrams, R. A., \& Zacks, J. M. (2014). Limits on action priming by pictures of objects. Journal of Experimental Psychology: Human Perception and Performance, 40(5), 1861-1873. https://doi.org/10.1037/a0037397 


\section{Figure Captions}

Figure 1. Procedure of an experimental trial in the two priming conditions: neutral priming (left) vs. action priming (right). 


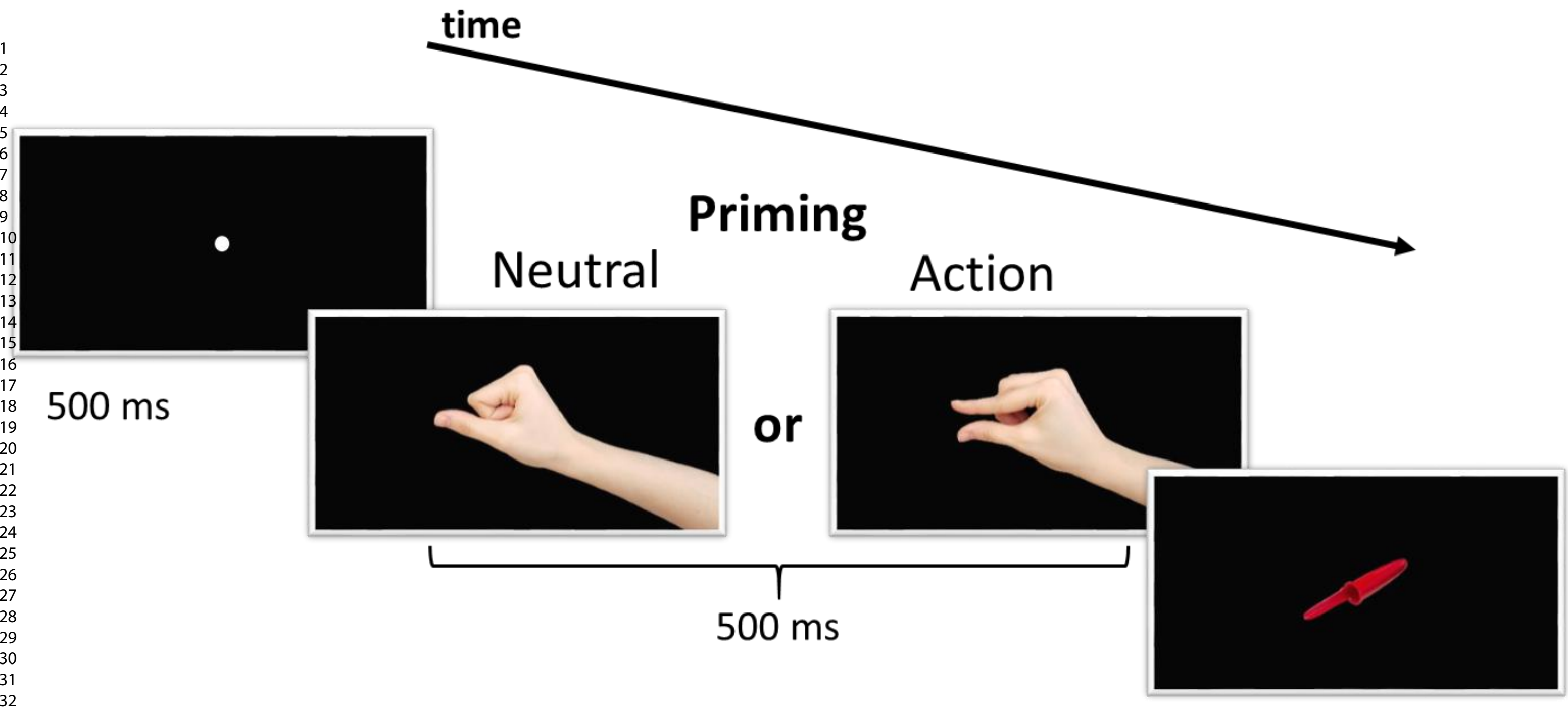

4 sec or until response

Manufactured or Natural ? 
Table 1. Mean RTs in milliseconds (SD) as a function of Category (Manufactured, Natural), Grasp Type (Power Grip, Precision Grip) and Priming (Action, Neutral).

\begin{tabular}{|c|c|c|c|c|c|c|c|}
\hline \multicolumn{4}{|c|}{ Manufactured } & \multicolumn{4}{|l|}{ Natural } \\
\hline \multicolumn{2}{|c|}{ Power Grip } & \multicolumn{2}{|c|}{ Precision Grip } & \multicolumn{2}{|c|}{ Power Grip } & \multicolumn{2}{|c|}{ Precision Grip } \\
\hline Action & Neutral & Action & Neutral & Action & Neutral & Action & Neutral \\
\hline 650.4 & 716.1 & 641.8 & 681.6 & 641.5 & 657.2 & 656 & 639.9 \\
\hline (139.2) & (170.1) & (120.2) & (201) & (176.3) & (182.9) & (162.7) & (115.2) \\
\hline
\end{tabular}

